Homology, Homotopy and Applications, vol.21(1), 2019, pp.49-71

\title{
THE CATEGORICAL SEQUENCE OF A RATIONAL SPACE
}

\author{
JULIENNE DARE HOUCK AND JEFFREY STROM
}

(communicated by John R. Klein)

\begin{abstract}
The categorical sequence of a space $X$ is a sequence of integers that encodes the growth of the Lusternik-Schnirelmann category of its CW skeleta as dimension increases. Restrictions on these sequences found in $[\mathbf{1 3}]$ have proven to be powerful tools in studying and computing L-S category, motivating the search for additional restrictions. In this paper we study the initial three-term segments of the categorical sequences of rational spaces of finite type. We show that there is another restriction: a sequence of the form $(a, b, a+b, \ldots)$ is the categorical sequence of a rational space of finite type if and only if $b \equiv 2 \bmod a-1$. With the possible exception of a small number of values of $c$ for each $a$, all other three-term initial sequences are realizable by simply-connected rational spaces of finite type.
\end{abstract}

\section{Introduction}

The Lusternik-Schnirelmann category of a map $f: X \rightarrow Y$ is the least integer $n$ for which there is an open cover $X=U_{0} \cup U_{1} \cup \cdots \cup U_{n}$ such that each restriction $\left.f\right|_{U_{i}}$ is nullhomotopic. This is a homotopy invariant of $f$, denoted cat $(f)$. The category of a map generalizes Lusternik and Schnirelmann's original concept of the category of a space, which we can define as $\operatorname{cat}(X)=\operatorname{cat}\left(\operatorname{id}_{X}\right)$. When $f: A \hookrightarrow X$ is the inclusion of a subspace, $\operatorname{cat}(f)$ is written $\operatorname{cat}_{X}(A)$ and called the category of $A$ in $X$.

It is easy to see that the category of a composition $f \circ g$ is bounded above by both cat $(f)$ and $\operatorname{cat}(g)$. Let us apply this observation to a CW complex $X$ with two CW decompositions $\left\{X_{n}\right\}$ and $\left\{X_{n}^{\prime}\right\}$. Since the inclusion $X_{j} \hookrightarrow X$ of the $j$-skeleton factors up to homotopy through the inclusion of the $k$-skeleton $X_{k}^{\prime} \hookrightarrow X$ when $j \leqslant k$, we have $\operatorname{cat}_{X}\left(X_{n}\right) \leqslant \operatorname{cat}_{X}\left(X_{n}^{\prime}\right)$ and vice versa; thus the list

$$
\operatorname{cat}_{X}\left(X_{1}\right), \operatorname{cat}_{X}\left(X_{2}\right), \ldots, \operatorname{cat}_{X}\left(X_{n}\right), \ldots
$$

is a weakly increasing sequence that depends only on the homotopy type of $X$. The categorical sequence of $X$ is the sequence $\sigma_{X}: \mathbb{N} \rightarrow \mathbb{N} \cup\{\infty\}$ defined by

$$
\sigma_{X}(k)=\inf \left\{n \mid \operatorname{cat}_{X}\left(X_{n}\right) \geqslant k\right\}
$$

(with the standard convention that $\inf (\varnothing)=\infty$ and the unfortunately nonstandard

Received January 12, 2018; published on August 22, 2018.

2010 Mathematics Subject Classification: 55M30, 55P62, 55Q15.

Key words and phrases: Lusternik-Schnirelmann category, rational homotopy theory.

Article available at http://dx.doi.org/10.4310/HHA.2019.v21.n1.a3

Copyright (c) 2018, International Press. Permission to copy for private use granted. 
convention that $0 \notin \mathbb{N}$ ); it records the dimensions $n$ at which the category of the skeleta jumps from $k-1$ to $k$. The main theorem of [13] establishes the following principal properties of categorical sequences:

1. $\sigma_{X}$ is superadditive:

$$
\sigma_{X}\left(k_{1}+k_{2}+\cdots+k_{r}\right) \geqslant \sigma_{X}\left(k_{1}\right)+\sigma_{X}\left(k_{2}\right)+\cdots+\sigma_{X}\left(k_{r}\right),
$$

for any $k_{1}, k_{2}, \ldots, k_{r} \in \mathbb{N}$, and

2. if $X$ is simply-connected and equality holds in (1) for some $k_{1}, k_{2}, \ldots, k_{r}$, then (writing $n_{i}=\sigma_{X}\left(k_{i}\right)$ ) the exterior cup product map

$$
\widetilde{H}^{n_{1}}\left(X ; G_{1}\right) \otimes \cdots \otimes \widetilde{H}^{n_{r}}\left(X ; G_{r}\right) \rightarrow \widetilde{H}^{n_{1}+\cdots+n_{r}}\left(X ; G_{1} \otimes \cdots \otimes G_{r}\right)
$$

is nonzero for some choice of coefficient groups $G_{1}, \ldots, G_{r}$.

Statement (1) shows that not every increasing sequence can be the categorical sequence of a space. Statement (2) already has useful content in the case $r=1$ : it implies that if $\sigma_{X}(k)=n$ then $\widetilde{H}^{n}(X ; G) \neq 0$ for some abelian group $G$.

Restrictions on categorical sequences can be powerful tools for computation. For example, it is easy to show, using the two properties listed above, that any simplyconnected space $X$ having $H^{*}(X ; \mathbb{Z}) \cong \Lambda_{\mathbb{Z}}\left(x_{3}, x_{7}, x_{11}\right)$ must have cat $(X) \leqslant 5$. It is explained in [13] that this inequality implies a result of Ghienne [9]: every space in the Mislin genus of $S p(3)$ has L-S category equal to 5. For another example, consider the following question asked by Dranishnikov, Katz, and Rudyak [5]: if $M$ is an $n$-dimensional manifold with $\pi_{1}(M)$ a free group, must it be that cat $(M) \leqslant n-2$ ? Any CW complex $M$ with $\pi_{1}(M)$ free has a CW decomposition with $M_{2} \simeq\left(\bigvee S^{1}\right) \vee$ $\left(\bigvee S^{2}\right)$, which has L-S category (at most) 1 . Therefore $\sigma_{M}(2) \geqslant 3$ and the superadditivity property (1) implies $\sigma_{M}(2 k) \geqslant 3 k$, forcing cat $(M)$ to be bounded above by roughly $\frac{2}{3} \operatorname{dim}(M)$. (This result appeared in [16] and has since been significantly improved and extended by Dranishnikov [11].)

In this paper we show that there is an additional condition satisfied by the first three values of the categorical sequences of rational spaces of finite type.

Theorem 1.1. Let $a, b, c \in \mathbb{N}$ with $a \geqslant 2$ and $b \geqslant 2 a$. Then there is a rational space $X$ of finite type having

$$
\sigma_{X}=(a, b, a+b, \ldots)
$$

if and only if $b \equiv 2 \bmod a-1$.

It is natural to ask whether other three-term initial sequences not excluded by Theorem 1.1 can be realized as categorical sequences.

Theorem 1.2. Let $a, b, c \in \mathbb{N}$ with $a \geqslant 2$ and $b \geqslant 2 a$. If the equation $s(a-1)+t a+$ $2=c$ can be solved with $s, t \in \mathbb{N}$ (so that $s, t>0$ ), then for every $b$ with $2 a \leqslant b<c-a$ there is a simply-connected rational space $X$ of finite type whose categorical sequence has the form $\sigma_{X}=(a, b, c, \ldots)$.

We conclude this introduction with a few remarks and observations:

1. Theorem 1.1 does not apply to the categorical sequences of nonrational spaces. For example, it follows from [15, Ex. 4.4] that for all $n \geqslant 2$, the categorical 
sequence of the symplectic group $S p(n)$ has the form $\sigma_{S p(n)}=(3,7,10, \ldots)$, which by Theorem 1.1 cannot be the categorical sequence of a rational space of finite type.

2. Theorem 1.2 implies that for $a=2$ or 3 , every sequence not excluded by Theorem 1.1 is actually realized as the categorical sequence of some simply-connected rational space of finite type.

3. Theorems 1.1 and 1.2 leave finitely many realization questions open for each $a>3$. Sequences $(a, b, c, \ldots)$ become easier to realize as $c-b$ increases. For $3<a \leqslant 6$ the situation for sequences of the form $(a, b, a+b+1, \ldots)$ is as follows:

(a) We can show 'by hand' that $X=P_{14}\left(S^{6} \vee S^{7}\right)$ has $\sigma_{X}=(6,15,22, \ldots)$ and that $Y=P_{24}\left(S^{6} \vee S^{7}\right)$ has $\sigma_{Y}=(6,25,32, \ldots)$. (The notation $P_{n}()$ indicates a Postnikov section; see Section 2.1 for our indexing convention.)

(b) Knowing this, for $a=4,5,6$, the only three-term initial sequences whose realizability is yet to be resolved are

$$
\begin{array}{lll}
(4,9,14, \ldots) & (5,11,17, \ldots) & (6,13,20, \ldots) \\
& (5,12,18, \ldots) & (6,14,21, \ldots) \\
& (5,16,22, \ldots) & (6,19,26, \ldots) \\
& & (6,20,27, \ldots)
\end{array}
$$

\section{Acknowledgments}

Our ideas about these questions were shaped by conversations with Don Stanley, John Oprea, and Martin Arkowitz. We are grateful to Bruno Le Floch for providing (via MathOverflow) the idea for the argument presented in Section 5.1.

\section{Preliminaries}

\subsection{Basics}

We refer to $[\mathbf{1 9}]$ for basic terminology and results on homotopy theory. We write $\operatorname{conn}(X)=n-1$ to indicate that $X$ is $(n-1)$-connected but not $n$-connected. Postnikov approximations and connective covers play key roles in our work. We write $p_{n}: X \rightarrow P_{n}(X)$ for the $n^{\text {th }}$ Postnikov approximation, meaning that $\pi_{k}\left(P_{n}(X)\right)=0$ for $k \geqslant n$ and $p_{n}$ induces isomorphisms on $\pi_{k}$ for $k<n$. The homotopy fiber of $p_{n}$ is the $(n-1)$-connective cover of $X$, denoted $X\langle n\rangle \rightarrow X$. On the domain side, $X_{n}$ denotes the $n$-skeleton of a CW complex $X$. We write $A^{T}$ for the transpose of the matrix $A$.

In this paper we are only concerned with simply-connected spaces of finite rational type, though some of our supporting results hold more generally.

\subsection{Lusternik-Schnirelmann category}

We refer the reader to the book [4] and the survey article [11] for background and information about the standard theory of L-S category.

Categorical sequences A detailed study of the L-S category of the CW skeleta of a CW complex is made in [13] using categorical sequences. The categorical 
sequence of a CW complex $X$ is the function $\sigma_{X}: \mathbb{N} \rightarrow \mathbb{N} \cup\{\infty\}$ defined by

$$
\sigma_{X}(k)=\inf \left\{n \mid \operatorname{cat}_{X}\left(X_{n}\right) \geqslant k\right\} .
$$

Notice that $\sigma_{X}(1)=1+\operatorname{conn}(X)$, so $\sigma_{X}(1)>1$ if and only if $X$ is simply-connected. It is proved in $[\mathbf{1 3}]$ that if $n>\operatorname{conn}(X)$ then $\operatorname{cat}_{X}\left(X_{n}\right)=\operatorname{cat}\left(X_{n}\right)$. In fact, this result has a useful generalization, established in [10, Lemma 4.2.2].

Proposition 2.1. Suppose $\operatorname{conn}(Y)=\operatorname{conn}(P)=a-1$ and let $f: Y \rightarrow P$ be an $n$ equivalence with $n \geqslant a$. If

$$
\operatorname{cat}(f) \geqslant \frac{\operatorname{dim}(Y)-(n-1)}{a}
$$

then $\operatorname{cat}(f)=\operatorname{cat}(Y)$.

Proof. For any function $f: Y \rightarrow P$ we have $\operatorname{cat}(f)=\operatorname{cat}\left(f \circ \operatorname{id}_{Y}\right) \leqslant \operatorname{cat}\left(\operatorname{id}_{Y}\right)=$ $\operatorname{cat}(Y)$, so we only have to show that $\operatorname{cat}(Y) \leqslant \operatorname{cat}(f)$. Write $\operatorname{cat}(f)=k$.

Let $G_{k}(Y) \rightarrow Y$ and $G_{k}(P) \rightarrow P$ be the $k^{\text {th }}$ Ganea fibrations of $Y$ and $P$, respectively. The statements cat $(Y) \leqslant k$ and $\operatorname{cat}(f) \leqslant k$ are equivalent to the existence of solutions to the lifting problems

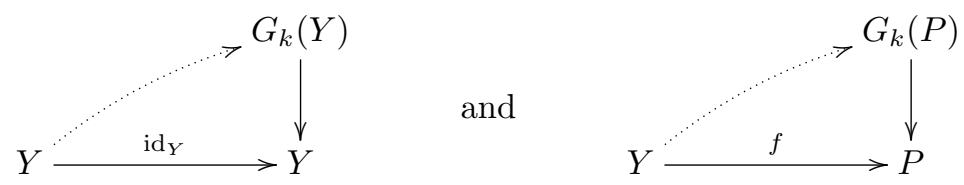

respectively $[\mathbf{4}, \mathbf{8}]$. Let $p: Q \rightarrow Y$ be the pullback of $G_{k}(P) \rightarrow P$ by $f$ and construct the diagram

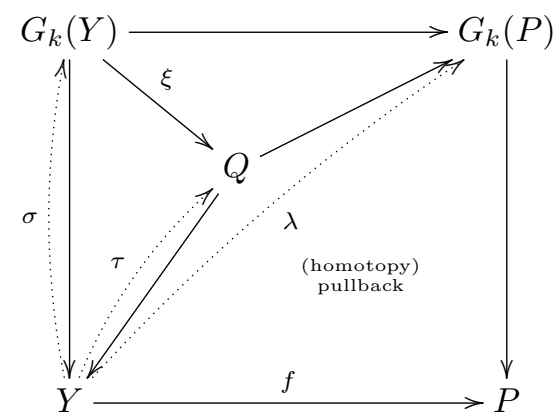

The homotopy fiber of the comparison map $\xi$ is the same as the homotopy fiber of the map $F_{k}(Y) \rightarrow F_{k}(P)$ between the fibers of the vertical maps [3, Lemma 2.1]. According to Ganea $[\mathbf{4}, \mathbf{8}]$, this map may be identified with the $(k+1)$-fold join $\Omega(f)^{*(k+1)}$. Since $f$ is an $n$-equivalence and $\operatorname{conn}(Y)=\operatorname{conn}(P)=a-1, \Omega(f)^{*(k+1)}$ is a $(k a+n-1)$-equivalence. Since $\xi$ and $\Omega(f)^{*(k+1)}$ have the same homotopy fiber, $\xi$ is a $(k a+n-1)$-equivalence as well.

Now cat $(f) \leqslant k$ gives a lift $\lambda: Y \rightarrow G_{k}(P)$ and the pullback property allows us to derive a section $\tau: Y \rightarrow Q$. Since

$$
k=\operatorname{cat}(f) \geqslant \frac{\operatorname{dim}(Y)-(n-1)}{a},
$$

the induced map $\xi_{*}:\left[Y, G_{k}(Y)\right] \rightarrow[Y, Q]$ is surjective, so the section $\tau$ lifts to a section $\sigma: Y \rightarrow G_{k}(Y)$. It follows that $\operatorname{cat}(Y) \leqslant k$. 
CATEGory WEIGHT The category weight of a nonzero cohomology class $u \in$ $\widetilde{H}^{*}(X)$ (with any coefficients) is the greatest integer $n$ for which $f^{*}(u)=0$ for all maps $f: Z \rightarrow X$ with cat $(f)<n$; it is denoted $\operatorname{wgt}(u)$. Clearly cat $(f) \geqslant n$ if there is a class $u \in \widetilde{H}^{*}(X)$ with $\operatorname{wgt}(u)=n$ and $f^{*}(u) \neq 0$. A version of this invariant was introduced by Fadell and Husseini in $[\mathbf{6}]$ and explored in detail (and modified for homotopy invariance) in $[\mathbf{1 4}, \mathbf{1 7}, \mathbf{1 8}]$.

It is easy to see that if $f: X \rightarrow Y, u \in \widetilde{H}^{*}(Y)$ and $f^{*}(u) \neq 0$ then $\operatorname{wgt}\left(f^{*}(u)\right) \geqslant$ $\operatorname{wgt}(u)$. Category weight was invented to estimate the category of spaces, but it can also be used to obtain more delicate information best expressed in terms of categorical sequences.

Proposition 2.2. If $u \neq 0 \in \widetilde{H}^{n}(X)$ with $\operatorname{wgt}(u)=k$, then $\sigma_{X}(k) \leqslant n$.

Proof. Since $i_{n}: X_{n} \hookrightarrow X$ induces an injection on $\widetilde{H}^{n}, i_{n}^{*}(u) \neq 0$ and, consequently, $\operatorname{cat}_{X}\left(X_{n}\right)=\operatorname{cat}\left(X_{n}\right) \geqslant \operatorname{wgt}\left(i_{n}^{*}(u)\right) \geqslant \operatorname{wgt}(u)=k$.

One of the most important features of category weight is its behavior with respect to cup products $[\mathbf{1 4}, \mathbf{1 7}, \mathbf{1 8}]$.

Theorem 2.3. If $u, v \in \widetilde{H}^{*}(X)$ with $u v \neq 0$, then $\operatorname{wgt}(u v) \geqslant \operatorname{wgt}(u)+\operatorname{wgt}(v)$.

\subsection{Whitehead products and Samelson products}

The Whitehead product of the summand inclusions $\mathrm{in}_{1}: S^{n_{1}} \hookrightarrow S^{n_{1}} \vee S^{n_{2}}$ and $\operatorname{in}_{2}: S^{n_{2}} \hookrightarrow S^{n_{1}} \vee S^{n_{2}}$ is the homotopy class of a map $w: S^{n_{1}+n_{2}-1} \rightarrow S^{n_{1}} \vee S^{n_{2}}$ such that

$$
S^{n_{1}+n_{2}-1} \stackrel{w}{\longrightarrow} S^{n_{1}} \vee S^{n_{2}} \stackrel{i}{\longrightarrow} S^{n_{1}} \times S^{n_{2}}
$$

is a cofiber sequence. The orientation conventions for spheres specify which of two possible maps $w$ is. In general, the Whitehead product is the natural operation

$$
[,]: \pi_{n_{1}}(X) \otimes \pi_{n_{2}}(X) \rightarrow \pi_{n_{1}+n_{2}-1}(X)
$$

defined by the formula $[\alpha, \beta]=(\alpha, \beta) \circ w$, where $(\alpha, \beta): S^{n_{1}} \vee S^{n_{2}} \rightarrow X$ is the map which restricts to $\alpha$ on the first summand and $\beta$ on the second.

The homotopy groups $\pi_{*}(X)$ can be filtered by product length with respect to the Whitehead product. Thus we write

$$
\ell(\alpha)=\max \{n \mid \alpha \in \operatorname{span}\{n \text {-fold Whitehead products }\}\} .
$$

There is a related product in the homotopy groups of a loop space, called the Samelson product, which is induced by the commutator map $\Omega X \times \Omega X \rightarrow \Omega X$ and is denoted

$$
\langle,\rangle: \pi_{n_{1}-1}(\Omega X) \otimes \pi_{n_{2}-1}(\Omega X) \rightarrow \pi_{\left(n_{1}-1\right)+\left(n_{2}-1\right)}(\Omega X) .
$$

The canonical isomorphism $\lambda: \pi_{n}(X) \rightarrow \pi_{n-1}(\Omega X)$ relates these two products by the formula

$$
\lambda([\alpha, \beta])=-(-1)^{|\alpha|}\langle\lambda(\alpha), \lambda(\beta)\rangle .
$$

Theorem 2.4. The Samelson product gives $\pi_{*}(\Omega X)$ the structure of a graded Lie algebra. Consequently, the Whitehead product satisfies the formulas: 
- $(-1)^{|\alpha||\gamma|}[[\alpha, \beta], \gamma]+(-1)^{|\beta||\alpha|}[[\beta, \gamma], \alpha]+(-1)^{|\gamma||\beta|}[[\gamma, \alpha], \beta]=0$,

- $[\alpha, \beta]=(-1)^{|\alpha||\beta|}[\beta, \alpha]$.

An exposition of these results can be found in $[\mathbf{1 9}, \mathrm{Ch} . \mathrm{X}]$.

\subsection{Sullivan models for rational spaces}

The homotopy theory of simply connected rational spaces of finite type is modelled by their Sullivan minimal models. We will circumvent the definition and instead give a description of the main properties that we will need. A detailed exposition can be found in [7].

The Sullivan model for a simply-connected rational space $X$ of finite type is a commutative differential graded algebra (CDGA) over $\mathbb{Q}$ having the form $(\Lambda(V), d)$, where $V$ is a simply-connected graded vector space (i.e., $V^{n}=0$ for $n \leqslant 1$ ) of finite type and the differential $d$ satisfies $d(V) \subseteq \Lambda^{\geqslant 2}(V)$. The Sullivan model computes the rational cohomology of the space $X$, i.e., there is a natural isomorphism $H(\Lambda(V), d) \cong$ $H^{*}(X ; \mathbb{Q})$. There is a natural nondegenerate pairing

$$
\langle;\rangle: V \otimes_{\mathbb{Q}} \pi_{*}(X) \rightarrow \mathbb{Q}
$$

which establishes a natural ${ }^{1}$ isomorphism $V \cong \operatorname{Hom}_{\mathbb{Q}}\left(\pi_{*}(X), \mathbb{Q}\right)$. This canonical isomorphism enables us to convert a basis $\mathcal{B}$ for $\pi_{*}(X)$ to a dual basis $\mathcal{B}^{*}$ for $V$, and $\mathcal{B}^{*}$ will generate $\Lambda(V)$ as an algebra.

To evaluate $\left\langle\beta_{i}^{*} ; \alpha\right\rangle$ where $\beta_{i} \in \mathcal{B}$ and $\alpha \in \pi_{*}(X)$, write $\alpha=\sum_{j} c_{j} \beta_{j}$ with each $c_{j} \in \mathbb{Q}$ and compute

$$
\left\langle\beta_{i}^{*}, \alpha\right\rangle=\left\langle\beta_{i}^{*}, \sum_{j} c_{j} \beta_{j}\right\rangle=\sum_{j} c_{j}\left\langle\beta_{i}^{*}, \beta_{j}\right\rangle=c_{i} .
$$

The differential $d$ decomposes as a sum $d=d_{1}+d_{2}+\cdots$, where $d_{j}$ raises product length by $j$. The differential $d_{1}$ can be usefully described using a second bracket

$$
\langle;,\rangle: \Lambda^{2}(X) \otimes_{\mathbb{Q}} \pi_{*}(X) \otimes_{\mathbb{Q}} \pi_{*}(X) \rightarrow \mathbb{Q}
$$

defined in terms of the first bracket by the formula

$$
\langle v \cdot w ; \alpha, \beta\rangle=\langle v ; \beta\rangle\langle w ; \alpha\rangle+(-1)^{|w||\alpha|}\langle v ; \alpha\rangle\langle w ; \beta\rangle .
$$

Now $d_{1}$ is characterized by the formula

$$
\begin{aligned}
\left\langle d_{1}(v) ; \alpha, \beta\right\rangle & =(-1)^{|v|}\langle v ;[\alpha, \beta]\rangle \\
& =(-1)^{|\alpha|+|\beta|-1}\langle v ;[\alpha, \beta]\rangle,
\end{aligned}
$$

where $v \in V$ (the two sign formulas agree because $\langle v ;[\alpha, \beta]\rangle$ can be nonzero only if $|v|=|[\alpha, \beta]|)[7]$. To see that this formula determines $d_{1}$, first observe that since $d_{1}$ is a derivation, it suffices to determine $d_{1}$ on the generating set $\mathcal{B}^{*}$. If $\beta^{*} \in \mathcal{B}^{*}$ then $d_{1}\left(\beta^{*}\right) \subseteq \Lambda^{2}(V)$. Write $\mathcal{B}^{*}=\left\{\beta_{u} \mid u \in \mathcal{U}\right\}$ and impose a linear order on the index set $\mathcal{U}$; then

\footnotetext{
${ }^{1} \mathrm{~A}$ map of spaces induces a map of Sullivan minimal models, but not naturally; nevertheless, its homology $H(\Lambda(V), d)$ is functorial, and the graded vector space $V$ is functorial on the homotopy category of simply-connected rational spaces of finite type when identified with $\Lambda^{\geqslant 1}(V) / \Lambda^{\geqslant 2}(V)$.
} 
$\left(\mathcal{B}^{*}\right)^{(2)}=\left\{\beta_{u}^{*} \beta_{v}^{*} \mid\right.$ for $u<v$ and for $u=v$ when $\left|\beta_{u}\right|$ is even $\}$

is a basis for $\Lambda^{2}(V)$. Then we can write

$$
d_{1}\left(\beta^{*}\right)=\sum_{\left(\mathcal{B}^{*}\right)^{(2)}} c_{u, v} \beta_{u}^{*} \beta_{v}^{*} \quad \text { with } c_{u, v} \in \mathbb{Q} .
$$

The following proposition shows explicitly how the second bracket determines the coefficients $c_{u, v}$.

Proposition 2.5. Let $X$ be a simply-connected rational space of finite type with Sullivan minimal model $(\Lambda(V), d)$, and let $\beta^{*} \in V$ correspond to $\beta \in \pi_{*}(X)$ under the canonical isomorphism $V \cong \operatorname{Hom}_{\mathbb{Q}}\left(\pi_{*}(X), \mathbb{Q}\right)$. The coefficient $c_{i, j}$ in the formula

$$
d_{1}\left(\beta^{*}\right)=\sum_{\left(\mathcal{B}^{*}\right)^{(2)}} c_{u, v} \beta_{u}^{*} \beta_{v}^{*}
$$

is given by

$$
\begin{aligned}
c_{i, j} & =(-1)^{\left(\left|\beta_{i}\right|-1\right)\left(\left|\beta_{j}\right|-1\right)}\left\langle\beta^{*},\left[\beta_{i}, \beta_{j}\right]\right\rangle \quad \text { if } i<j \in \mathcal{U}, \text { and } \\
c_{i, i} & =-\frac{1}{2}\left\langle\beta^{*},\left[\beta_{i}, \beta_{i}\right]\right\rangle .
\end{aligned}
$$

Proof. If $i<j$, we have

$$
\begin{aligned}
\left\langle d_{1}\left(\beta^{*}\right) ; \beta_{i}, \beta_{j}\right\rangle & =\left\langle\sum c_{u, v} \beta_{u}^{*} \beta_{v}^{*} ; \beta_{i}, \beta_{j}\right\rangle \\
& =\sum c_{u, v}\left\langle\beta_{u}^{*} \beta_{v}^{*} ; \beta_{i}, \beta_{j}\right\rangle \\
& =\sum c_{u, v}\left(\left\langle\beta_{u}^{*} ; \beta_{j}\right\rangle\left\langle\beta_{v}^{*} ; \beta_{i}\right\rangle+(-1)^{\left|\beta_{v}^{*}\right|\left|\beta_{i}\right|}\left\langle\beta_{u}^{*} ; \beta_{i}\right\rangle\left\langle\beta_{v}^{*} ; \beta_{j}\right\rangle\right) \\
& =(-1)^{\left|\beta_{v}^{*}\right|\left|\beta_{i}\right|} c_{i, j}
\end{aligned}
$$

since $u \leqslant v$ for each term in the sum. This can only be nonzero if $v=j$, so we can write

$$
\left\langle d_{1}\left(\beta^{*}\right) ; \beta_{i}, \beta_{j}\right\rangle=(-1)^{\left|\beta_{j}^{*}\right|\left|\beta_{i}\right|} c_{i, j} .
$$

On the other hand $\left\langle d_{1}\left(\beta^{*}\right) ; \beta_{i}, \beta_{j}\right\rangle=(-1)^{\left|\beta_{i}\right|+\left|\beta_{j}\right|-1}\left\langle\beta^{*} ;\left[\beta_{i}, \beta_{j}\right]\right\rangle$ so

$$
c_{i, j}=(-1)^{\left(\left|\beta_{i}\right|-1\right)\left(\mid \beta_{j}-1\right)}\left\langle\beta^{*} ;\left[\beta_{i}, \beta_{j}\right]\right\rangle
$$

as desired. A similar computation gives $c_{i, i}=-\frac{1}{2}\left\langle\beta^{*},\left[\beta_{i}, \beta_{i}\right]\right\rangle$.

\subsection{Category weight for rational spaces}

There is a rich theory of the L-S category of rational spaces, almost none of which is needed for our present purposes. We only need to be able to recognize the category weight of a class in $\widetilde{H}^{n}(X ; \mathbb{Q})$ in terms of its representatives in the Sullivan model $(\Lambda(V), d)$ for $X$.

Proposition 2.6. If a class $u \in H(\Lambda(V), d) \cong H^{*}(X ; \mathbb{Q})$ is represented by an element $z \in \Lambda^{\geqslant k}(V)$, then $\operatorname{wgt}(u) \geqslant k$.

Proof. This is explained in the discussion following Definition 5.2.2 in [4]. 


\section{Reduction to canonical examples}

In this section we show that in our search for rational spaces whose categorical sequence has the form $(a, b, a+b, \ldots)$ we can restrict our attention to Postnikov sections of wedges of rational spheres.

Theorem 3.1. Let $a \geqslant 2$ and suppose there is a rational space of finite type whose categorical sequence has the form $(a, b, a+b, \ldots)$. Then there is a finite wedge of rational spheres $W$ with $\operatorname{conn}(W)=a-1$ and $\operatorname{dim}(W) \leqslant b-2$ such that the categorical sequence of the Postnikov section $P=P_{b-1}(W)$ has the form $\sigma_{P}=(a, b, a+b, \ldots)$.

Proof. Suppose $\sigma_{X}=(a, b, a+b, \ldots)$. Since $\sigma_{X}(2)=b$, $\operatorname{cat}\left(X_{b-1}\right)=1$, so $X_{b-1}$ has the homotopy type of a wedge $\bar{W}$ of rational spheres; write $\bar{W}=W \vee \bigvee S^{b-1}$, with $\operatorname{dim}(W) \leqslant b-2$. Then both $W \hookrightarrow \bar{W}$ and $\bar{W} \rightarrow X$ induce isomorphisms on $\pi_{k}$ for all $k<b-1$, which implies that the induced maps

$$
P_{b-1}(W) \rightarrow P_{b-1}(\bar{W}) \rightarrow P_{b-1}(X)
$$

are (weak) homotopy equivalences. Write $P=P_{b-1}(W)$; we show that $\sigma_{X}(k)=\sigma_{P}(k)$ for $k \leqslant 3$.

First, $\sigma_{P}(1)=\operatorname{conn}(P)+1=\operatorname{conn}(X)+1=a$ since $a \geqslant 2$ implies $a<2 a-1 \leqslant$ $b-1$. Since $P$ can be constructed to have $P_{b-1}=X_{b-1}$ which has L-S category 1 , $\sigma_{P}(2)>b-1$. By superadditivity, $\sigma_{P}(3) \geqslant \sigma_{P}(2)+\sigma_{P}(1) \geqslant b+a$.

We first determine $\sigma_{P}(2)$. For any $n \geqslant b$, all of the obstructions to solving the extension problem

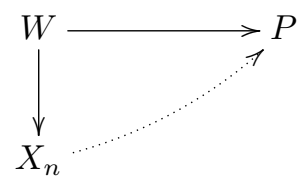

lie in zero groups, so a solution can be found, and any solution must be a $(b-1)$ equivalence. So choose a $(b-1)$-equivalence $f: X_{b} \rightarrow P$ extending $W \rightarrow P$. Any such map necessarily has

$$
\operatorname{cat}(f) \geqslant 1 \geqslant \frac{b-(b-1)}{a}=\frac{1}{a},
$$

so we can apply Proposition 2.1 to conclude cat $(f)=\operatorname{cat}\left(X_{b}\right)=2$. Since $f$ factors (up to homotopy) through $P_{b}$ by cellular approximation, we have $\operatorname{cat}\left(P_{b}\right) \geqslant \operatorname{cat}(f)=2$ and hence $\sigma_{P}(2) \leqslant b$. Since we also have $\sigma_{P}(2) \geqslant b$ we obtain $\sigma_{P}(2)=b$.

Next choose a $(b-1)$-equivalence $g: X_{a+b} \rightarrow P$ extending $f$; we have just seen that its restriction $\left.g\right|_{X_{b}}$ has L-S category 2. Therefore

$$
\operatorname{cat}(g) \geqslant 2 \geqslant \frac{(a+b)-(b-1)}{a}=\frac{a+1}{a},
$$

and Proposition 2.1 implies that

$$
\operatorname{cat}(g)=\operatorname{cat}\left(X_{a+b}\right)=3 .
$$

Since $g$ factors (up to homotopy) through $P_{a+b}$, it follows that $\operatorname{cat}\left(P_{a+b}\right) \geqslant 3$. This forces $\sigma_{P}(3)=a+b$ and completes the proof. 


\section{The Sullivan model for a wedge of spheres}

It is now clear that we need to study wedges of rational spheres and their Postnikov sections. For the entire section we will use the notation $W=\bigvee_{i \in \mathcal{I}} S^{n_{i}}$ to denote a fixed finite wedge of rational spheres with $n_{i} \geqslant 2$ for all $i \in \mathcal{I}$. Fix an ordered basis $\mathcal{B}=\left\{\beta_{u} \mid u \in \mathcal{U}\right\}$ for $\pi_{*}(W)$ such that

- $u \leqslant v$ implies $\ell\left(\beta_{u}\right) \leqslant \ell\left(\beta_{v}\right)$, and

- each $\beta_{u}$ is a Whitehead product monomial ${ }^{2}$ in the generators $x_{i} \in \pi_{n_{i}}(W)$ represented by the inclusions $\operatorname{in}_{i}: S^{n_{i}} \hookrightarrow W$.

Write $V=\operatorname{Hom}_{\mathbb{Q}}\left(\pi_{*}(W), \mathbb{Q}\right)$; then we may write the minimal model for $W$ in the form $(\Lambda(V), d)$.

Since we are studying rational homotopy in this section, all the algebra is done over the rationals. That is, $\otimes$ stands for $\otimes_{\mathbb{Q}}$, all cohomology is calculated using coefficients in $\mathbb{Q}$, and so on.

\subsection{Whitehead products and the homotopy groups of a wedge}

The inclusions of the summands represent classes $\beta_{i} \in \pi_{n_{i}}(W)$ which, according to the Hilton-Milnor theorem, generate $\pi_{*}(W)$ under the Whitehead product.

Theorem 4.1. Let $W$ be a simply-connected finite wedge of rational spheres. Then

1. the Samelson product gives $\pi_{*}(\Omega X)$ the structure of the free graded Lie algebra on the graded vector space $\Sigma^{-1} H_{*}(W)$,

2. there is an algebra isomorphism $H_{*}(\Omega W) \cong T_{\mathbb{Q}}\left(\Sigma^{-1} H_{*}(W)\right)$ between the Pontrjagin algebra of $\Omega W$ and the indicated tensor algebra, and

3. the Hurewicz map $\pi_{*}(\Omega W) \rightarrow H_{*}(\Omega W)$ represents $H_{*}(\Omega W)$ as a universal enveloping algebra.

Proof. Part (1) is proved in [7, Thm. 24.5(iv)]. Part (2) is the Bott-Samelson theorem $[\mathbf{1}]$ and part (3) is the Milnor-Moore theorem [12].

\subsection{The Sullivan model for a wedge of spheres}

Our topological simplification - that we need only look at wedges of rational spheres and their Postnikov sections - gives a corresponding algebraic simplification.

Proposition 4.2. If $(\Lambda(V), d)$ is the Sullivan model for a finite-type wedge of spheres, then $d=d_{1}$.

Because of this, we can give a convenient description of the differential in terms of Whitehead products. To state it we need to keep track of the product length of our basis elements. Thus we write $L_{n} \subseteq L$ for the subspace spanned by the $n$-fold brackets in our chosen basis $\mathcal{B}$ and

$$
V_{n}=\operatorname{span}\left\{\beta^{*} \mid \beta \in \mathcal{B} \text { is an } n \text {-fold bracket }\right\} .
$$

We need some more bases to state our matrix representation result:

- $\left(\mathcal{B}^{*}\right)^{(2)}=\left\{\beta_{u}^{*} \beta_{v}^{*} \mid u<v\right.$ or $u=v$ and $\left|\beta_{u}^{*}\right|$ is even $\}$ is a basis for $\bigoplus_{i+j=n} V_{i} V_{j} \subseteq$ $\Lambda(V)$,

\footnotetext{
${ }^{2}$ Remember that the Whitehead product is not associative.
} 
- $\mathcal{B}_{L}=\lambda(\mathcal{B})$ is a basis for the graded Lie algebra $L=\pi_{*}(\Omega W)$,

- $\mathcal{B}_{L}^{(2)}=\left\{\lambda\left(\beta_{u}\right) \otimes \lambda\left(\beta_{v}\right) \mid u<v\right.$ or $u=v$ and $\left|\beta_{u}\right|$ is even $\}$ is a basis for a subspace $K \subseteq \bigoplus_{i \leqslant j} L_{i} \otimes L_{j}$.

The restriction of the Samelson product to $K$ defines a function $s: K \rightarrow L_{n}$

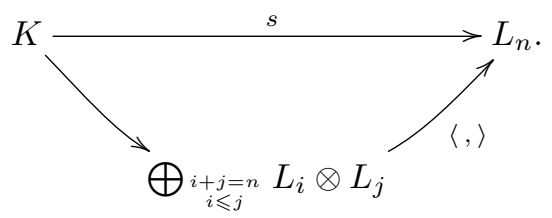

Let $M$ be the matrix representing $s$ with respect to the bases $\left(\mathcal{B}_{L}\right)^{(2)}$ and $\mathcal{B}_{L}$; say $M$ is a $p \times q$ matrix.

Proposition 4.3. Let $D$ be the matrix for the differential

$$
d: V_{n} \rightarrow \bigoplus_{\substack{i+j=n \\ i \leqslant j}} V_{i} V_{j}
$$

with respect to the dual bases $\mathcal{B}^{*}$ and $\left(\mathcal{B}^{*}\right)^{(2)}$. Let $Q$ be the $q \times q$ diagonal matrix with

- $(-1)^{\left(\left|\beta_{u}\right|-1\right)\left|\beta_{v}\right|}$ in the $\left(\beta_{u}, \beta_{v}\right)$ diagonal entry if $u<v$,

- $\frac{1}{2}$ in the $\left(\beta_{u}, \beta_{u}\right)$ diagonal entry if $\left|\beta_{u}\right|$ is even.

Then $D=Q M^{T}$.

Proof. Because $d=d_{1}$ in the Sullivan model for $W$ by Proposition 4.2, this follows from Proposition 2.5. The entry $d_{(u, v), w}$ in the $\left(\beta_{u}^{*}, \beta_{v}^{*}\right)$ row and $\beta_{w}^{*}$ column of $D$ is the coefficient of $\beta_{u}^{*} \beta_{v}^{*}$ in $d\left(\beta_{w}^{*}\right)$. According to Proposition 2.5, this is precisely (for $u \neq v)$

$$
d_{(u, v), w}=(-1)^{\left(\left|\beta_{u}\right|-1\right)\left(\left|\beta_{v}\right|-1\right)}\left\langle\beta_{w}^{*},\left[\beta_{u}, \beta_{v}\right]\right\rangle .
$$

On the other hand, the entry in the $\lambda\left(\beta_{u}\right) \otimes \lambda\left(\beta_{v}\right)$ column and $\lambda\left(\beta_{w}\right)$ row of $M$ is the unique $m_{w,(u, v)} \in \mathbb{Q}$ such that

$$
\left\langle\lambda\left(\beta_{u}\right), \lambda\left(\beta_{v}\right)\right\rangle=m_{w,(u, v)} \cdot \lambda\left(\beta_{w}\right)+\text { linearly independent terms. }
$$

Since

$$
\begin{aligned}
\lambda\left(\left[\beta_{u}, \beta_{v}\right]\right) & =-(-1)^{\left|\beta_{u}\right|}\left\langle\lambda\left(\beta_{u}\right), \lambda\left(\beta_{v}\right)\right\rangle \\
& =-(-1)^{\left|\beta_{u}\right|} s\left(\lambda\left(\beta_{u}\right) \otimes \lambda\left(\beta_{v}\right)\right) \\
& =-(-1)^{\left|\beta_{u}\right|} m_{w,(u, v)} \cdot \lambda\left(\beta_{w}\right)+\text { linearly independent terms }
\end{aligned}
$$

we have

$$
\left\langle\beta_{w}^{*},\left[\beta_{u}, \beta_{v}\right]\right\rangle=-(-1)^{\left|\beta_{u}\right|} m_{w,(u, v)} .
$$

Taken together, we have

$$
\begin{aligned}
d_{(u, v), w} & =(-1)^{\left(\left|\beta_{u}\right|-1\right)\left(\left|\beta_{v}\right|-1\right)}\left(-(-1)^{\left|\beta_{u}\right|} m_{w,(u, v)}\right) \\
& =(-1)^{\left(\left|\beta_{u}\right|-1\right)\left|\beta_{v}\right|} m_{w,(u, v)} .
\end{aligned}
$$

A similar computation, using the fact that $\left|\beta_{u}\right|$ must be even for there to be a $\lambda\left(\beta_{u}\right) \otimes \lambda\left(\beta_{u}\right)$ indexed column in $M$, yields the equation $d_{w,(u, u)}=\frac{1}{2} m_{(u, u), k}$. 


\subsection{Models for Postnikov sections}

If $(\Lambda(V), d)$ is the Sullivan model for a space $X$ of finite rational type, then the Postnikov section $X \rightarrow P_{n}(X)$ is modelled by the inclusion

$$
\left(\Lambda\left(V^{<n}\right),\left.d\right|_{\Lambda(V<n)}\right) \hookrightarrow(\Lambda(V), d),
$$

where $V^{<n}$ denotes the graded rational vector space with $V^{k}$ in dimension $k$ if $k<n$ and $V_{k}=0$ if $k \geqslant n$.

Proposition 4.4. Let $(\Lambda(V), d)$ be the Sullivan model for a simply-connected wedge of rational spheres $W$ with dimension at most $b-1$ (so $b \geqslant 3)$, and let $\left(\Lambda\left(V^{<b-1}\right)\right.$, $\left.\left.d\right|_{\Lambda(V<b-1)}\right)$ be the Sullivan model for $P_{b-1}(W)$. Then the set

$$
\left\{\left[d\left(\beta^{*}\right)\right]|\beta \in \mathcal{B},| \beta \mid=b-1\right\}
$$

is a basis for $H^{b}\left(P_{b-1}(W)\right)$.

Proof. Since $H^{b}(W)=0$ and $d=d_{1}$ increases product length by exactly 1 , the sequences

$$
\Lambda^{k}(V)^{b-1} \stackrel{d}{\rightarrow} \Lambda^{k+1}(V)^{b} \stackrel{d}{\rightarrow} \Lambda^{k+2}(V)^{b+1}, \quad k \geqslant 1
$$

in the Sullivan model for $W$ are exact. When we remove $V^{b-1}$ to form the model for $P_{b-1}(W)$, these sequences are unchanged except for the one with $k=1$, which becomes

$$
0 \stackrel{\widehat{d}}{\rightarrow} \Lambda^{2}(V)^{b} \stackrel{\widehat{d}}{\rightarrow} \Lambda^{3}(V)^{b+1}
$$

Therefore

$$
H^{b}\left(P_{b-1}(W)\right)=\operatorname{ker}(\widehat{d}) / \operatorname{im}(\widehat{d})=d\left(V^{b-1}\right) / 0 \cong d\left(V^{b-1}\right) .
$$

Since $H^{b-1}(W)=0$, the restriction of $d$ to $V^{b-1}$ is injective, so the basis $\left\{\beta^{*} \mid \beta \in \mathcal{B}\right.$, $|\beta|=b-1\}$ is carried by $d$ to the basis $\left\{d\left(\beta^{*}\right)|\beta \in \mathcal{B},| \beta \mid=b-1\right\}$ for $d\left(V^{b-1}\right)$, and the result follows.

4.4. Recognizing spaces realizing $(a, b, a+b, \ldots)$

We give criteria to recognize Postnikov sections $P$ of wedges of spheres with $\sigma_{P}=$ $(a, b, a+b, \ldots)$ from the ordinary algebraic properties of their cohomology algebras.

Proposition 4.5. Let $W$ be a simply-connected finite wedge of rational spheres with $\operatorname{conn}(W)=a-1$ and let $P=P_{b-1}(W) \quad(b \geqslant 2 a)$. Then every nonzero cohomology class $u \in \widetilde{H}^{\geqslant b}(P)$ has $\operatorname{wgt}(u) \geqslant 2$.

Proof. Since the Sullivan model for $P$ has no generators in dimensions $b-1$ or higher, every nonzero class $u \in \widetilde{H} \geqslant b(P)$ is represented by an element of $\Lambda^{\geqslant 2}\left(V^{<b-1}\right)$. Proposition 2.6 tells us $\operatorname{wgt}(u) \geqslant 2$.

Proposition 4.6. Let $W$ be a wedge of rational spheres with $\operatorname{conn}(W)=a-1$ and let $P=P_{b-1}(W)$. Then the following are equivalent:

1. $\sigma_{P}=(a, b, a+b, \ldots)$,

2. $\operatorname{cat}\left(P_{a+b}\right)=3$,

3. the cup product $\widetilde{H}^{a}(P) \otimes \widetilde{H}^{b}(P) \rightarrow \widetilde{H}^{a+b}(P)$ is nonzero, and 
4. there are $\alpha, \beta \in \mathcal{B}$ with $|\alpha|=a,|\beta|=b-1$ such that

$$
\left[\alpha^{*}\right] \cdot\left[d\left(\beta^{*}\right)\right] \neq 0 \in \widetilde{H}^{a+b}(P) .
$$

Proof. First observe that since the $(b-1)$-skeleton of $P$ is a wedge of rational spheres, $\sigma_{P}(2) \geqslant b$.

Now (1) implies (2) by definition and (2) implies (3) by the cup product property of categorical sequences. Proposition 4.4 shows that (3) implies (4). It remains to show that (4) implies (1). Given (4), Proposition 4.5 tells us that $\operatorname{wgt}\left(\left[d\left(\beta^{*}\right)\right]\right) \geqslant 2$. Therefore $\sigma_{P}(2)=b$ and $\sigma_{P}(3) \geqslant a+b$. Finally, $\operatorname{wgt}\left(\left[\alpha^{*}\right] \cdot\left[d\left(\beta^{*}\right)\right]\right) \geqslant 3$ by Theorem 2.3 , so $\sigma_{P}(3) \leqslant a+b$ by Proposition 2.2. Together with superadditivity and the inequality $\sigma_{P}(2) \geqslant b$, this implies (1).

\section{Rationally unrealizable spaces}

In this section we prove that if $b \not \equiv 2 \bmod a-1$, then no sequence of the form $(a, b, a+b, \ldots)$ can be realized as the categorical sequence of a rational space of finite type. To this end, we fix $a, b \in \mathbb{N}$ with $b \geqslant 2 a$ and $b \neq \equiv 2 \bmod a-1$. According to Theorem 3.1, if there were a space with categorical sequence $(a, b, a+b, \ldots)$, then such a space could be found having the form $P=P_{b-1}(W)$, where, as in the previous section, $W$ denotes a wedge $\bigvee_{j=1}^{k} S^{n_{j}}$ of rational spheres with $\operatorname{conn}(W)=a-1$ and $\operatorname{dim}(W)<b-1$. Therefore we fix such a $W$ and show that for any $\alpha, \beta \in \mathcal{B}$ with $|\alpha|=a$ and $|\beta|=b-1$, the class $\alpha^{*} \cdot d\left(\beta^{*}\right)$ is a boundary in the Sullivan model $\left(\Lambda\left(V^{<b-1}\right), d\right)$ for $P$ (it is obviously a boundary in the Sullivan model for $\left.W\right)$. Then Proposition 4.6 implies that $\sigma_{P}$ cannot have the form $(a, b, a+b, \ldots)$.

\subsection{A surjectivity condition}

Fix $n \geqslant 1$ and $r$ with $1 \leqslant r<k$ and write

$$
\begin{aligned}
U_{1}^{(r)} & =\operatorname{span}\left\{x_{1}^{*}, x_{2}^{*}, \ldots, x_{k}^{*}\right\}, \\
W_{n}^{(r)} & =\left\{\beta^{*} \mid \begin{array}{c}
\beta \in \mathcal{B}, \ell(\beta)=n, \text { and at least one } \\
\text { of } x_{r+1}, x_{r+2}, \ldots, x_{k} \text { is a factor of } \beta
\end{array}\right\},
\end{aligned}
$$

where $x_{i}^{*}$ is dual to the element $x_{i} \in \pi_{n_{i}}(W)$ represented by the inclusion $S^{n_{i}} \hookrightarrow W$. Thus $U_{1}^{(r)} \subseteq V_{1}$ and $W_{n}^{(r)} \subseteq V_{n}$ according to the notation introduced in Section 4.2. Since these subspaces are spanned by subsets of our preferred basis $\mathcal{B}^{*}$, they come equipped with canonical projection maps $V \rightarrow U_{1}^{(r)}$ and $V_{n} \rightarrow W_{n}^{(r)}$ (these projections are the identity on basis elements in common to the domain and target and zero on the other basis elements). We show in this section that the composition denoted $\rho$ in the diagram

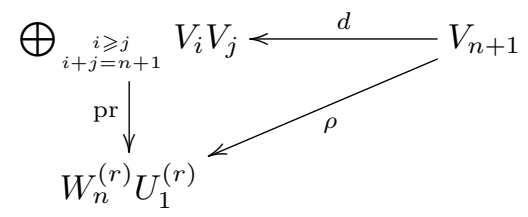

is surjective. 
If $D$ is the matrix representing $d$ in our chosen bases, then the matrix $R$ representing $\rho$ may be obtained from $D$ by selecting the rows indexed by $\beta^{*} x_{i}^{*}$ with $i \leqslant r$ and $\beta \in \mathcal{B}$ with at least one of $x_{r+1}, x_{r+2}, \ldots, x_{k}$ as a factor and $\ell(\beta)=n$.

Let $L=\pi_{*}(\Omega W)$ be the free graded Lie algebra on the generators $x_{1}, x_{2}, \ldots, x_{k}$, and let $L_{n}$ be the subspace spanned by the $n$-fold brackets. Let $U=\operatorname{span}\left\{x_{i} \mid i \leqslant r\right\}$ and let $W$ be the span of the $n$-fold brackets that involve at least one of $x_{r+1}, x_{r+2}$, $\ldots, x_{k}$. Define the map $\mathfrak{b}$ as the composition

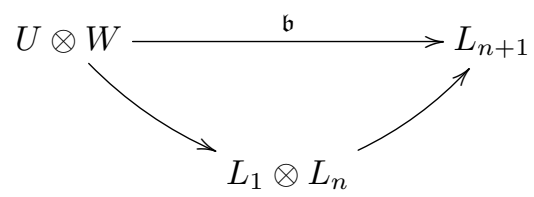

of the inclusion with the Lie bracket. In the notation of Proposition 4.3, the map $\mathfrak{b}$ is represented by the matrix $B$ obtained from $M$ by selecting the columns indexed by $\left[\beta, x_{i}\right]$ such that $i \leqslant r$ and $\beta \in \mathcal{B}$ with $\ell(\beta)=n$ and at least one of $x_{r+1}, x_{r+2}, \ldots, x_{k}$ as a factor. Thus Proposition 4.3 shows that $R$ is the product of $B^{T}$ with an invertible diagonal matrix (the matrix obtained from $Q$ with certain rows and the corresponding columns deleted). To show that $\rho$ is surjective, then, it suffices to show that $\mathfrak{b}$ is injective.

The Milnor-Moore and Bott-Samelson theorems (recorded above in Theorem 4.1) allow us to view the graded Lie algebra as a subspace of the tensor algebra $T\left(V_{1}\right)$ with respect to the standard graded commutator bracket $[x, y]=x y-(-1)^{|x||y|} y x$. Under this identification, $U$ remains the span of $x_{1}, x_{2}, \ldots, x_{r}$ and $W$ consists of (some but not all) noncommutative polynomials in $x_{1}, x_{2}, \ldots, x_{r}$ in which every monomial has precisely $n$ factors, at least one of which is in $\left\{x_{r+1}, \ldots, x_{k}\right\}$.

We show that $\mathfrak{b}$ has trivial kernel. Let $y=\sum_{i=1}^{r} x_{i} \otimes w_{i} \neq 0 \in U \otimes W$. Then

$$
\mathfrak{b}(y)=\sum_{i=1}^{r}\left(x_{i} w_{i}-(-1)^{\left|w_{i}\right|\left|x_{i}\right|} w_{i} x_{i}\right) \text {. }
$$

For a monomial $m=c x_{j_{1}} x_{j_{2}} \cdots x_{j_{m}} \in W_{n}^{(r)}$, write

$$
\mathcal{J}(m)=\max \left\{i \mid j_{i}>r\right\} .
$$

Choose one $w_{j}$ which contains as a summand a monomial $m$ for which $\mathcal{J}(m)$ is maximal among all the summands of all the $w_{i}$. Then

$$
\mathfrak{b}(y)=x_{j} m+\text { other terms }
$$

and the monomial $x_{j} m$ cannot be a summand of any of the other terms of $\mathfrak{b}(y)$. It is not a summand of $x_{i} w_{i}$ for any $i$ because $\mathcal{J}\left(x_{j} m\right)>\mathcal{J}\left(x_{i} m^{\prime}\right)$ for every other monomial summand $m^{\prime}$ of every $w_{i}$. We conclude that $\mathfrak{b}(y) \neq 0$ and hence that $\mathfrak{b}$ is injective.

\subsection{Proof of nonrealizability}

Now we prove that if $b \not \equiv 2 \bmod a-1$ then there is no simply-connected rational space $X$ of finite type whose categorical sequence has the form $\sigma_{X}=(a, b, a+b, \ldots)$. Let us assume to the contrary that there is such a space. By Theorem 3.1 we find 
such an example that has the form $P=P_{b-1}(W)$ where $W$ is a wedge of rational spheres. Proposition 4.6(4) ensures that there is a nonzero cup product of the form $\left[\alpha^{*}\right]\left[d\left(\beta^{*}\right)\right] \neq 0 \in \widetilde{H}^{a+b}(P)$ with $\alpha \in \mathcal{B}$ with $|\beta|=b-1$; write $n=\ell(\beta)$. We show that, on the contrary, all such cup products vanish.

Consider first the model $(\Lambda(V), d)$ for $W$. Write $V=\operatorname{span}\left\{x_{1}, x_{2}, \ldots, x_{k}\right\}$, where the generators have been labelled in weakly increasing order of dimension. Let $r$ be such that $x_{1}, \ldots, x_{r}$ have dimension $a$ and $x_{r+1}, \ldots, x_{k}$ have dimension strictly greater than $a$.

Now the congruence $b \not \equiv 2 \bmod a-1$ comes into play. The Whitehead product of elements in $\pi_{a}(W)$ lies in a dimension that is congruent to $1 \bmod a-1$. Since we assume $b \not \equiv 2 \bmod a-1$, we have

$$
|\beta|=b-1 \not \equiv 1 \bmod a-1,
$$

and so the Whitehead monomial $\beta$ must have at least one factor $x_{i}$ with $\left|x_{i}\right| \neq a$. Hence $r<k$.

Using the notation from Section 5.1, we write

$$
\bigoplus_{\substack{i \leqslant j \\ i+j=n+1}} V_{i} V_{j}=\left(U_{1}^{(r)} W_{n}^{(r)}\right) \oplus Y,
$$

where $Y=\operatorname{ker}(\mathrm{pr})$ is the span of members of the canonical basis not contained in $U_{1}^{(r)} W_{n}^{(r)}$. Since

$$
\operatorname{pr}\left(d\left(V_{n+1}\right)\right)=\rho\left(V_{n+1}\right)=U_{1}^{(r)} W_{n}^{(r)},
$$

we may choose $z \in d\left(V_{n+1}\right)$ such that $\operatorname{pr}(z)=\operatorname{pr}\left(\alpha^{*} \beta^{*}\right)$. Then $\alpha^{*} \beta^{*}-z \in \operatorname{ker}(d)=Y$. We will write $y=\alpha^{*} \beta^{*}-z$.

Now we move to the model $\left(\Lambda\left(V^{<b-1}\right), d\right)$ for $P$. By construction, $Y$ is the span of products $x \cdot v$ with $|x|>a$ and $|v|<b-1$, which implies that $Y \subseteq \Lambda\left(V^{<b-1}\right)$. In this model, therefore, we have

$$
d(y)=d\left(\alpha^{*} \beta^{*}-z\right)=d\left(\alpha^{*} \beta^{*}\right)-d(z)=\alpha^{*} d\left(\beta^{*}\right) .
$$

Thus $\left[\alpha^{*}\right] \cdot\left[d\left(\beta^{*}\right)\right]=0$ in $H^{b}(P)$. We deduce that $\sigma_{P} \neq(a, b, a+b, \ldots)$.

\section{Rational realization of sequences}

In this section we complete the proof of Theorem 1.1 and prove Theorem 1.2.

\subsection{The spectral sequence}

Fix an $(a-1)$-connected wedge $W$ of rational spheres with $\operatorname{dim}(W)<b-1$, write $P=P_{b-1}(W)$, form the fiber sequence $W\langle b-1\rangle \rightarrow W \rightarrow P$, and apply the LeraySerre spectral sequence. The crucial features of the spectral sequence are displayed in Figure 1, in which the labelled groups are

$$
\begin{aligned}
& A_{1}=E_{2}^{a, 0} \cong H^{a}(P ; \mathbb{Q}), \\
& A_{2}=E_{2}^{a+1,0} \cong H^{a+1}(P ; \mathbb{Q}), \\
& B_{1}=E_{2}^{0, b-1} \cong H^{b-1}(W\langle b-1\rangle ; \mathbb{Q}), \\
& B_{2}=E_{2}^{0, b} \cong H^{b}(W\langle b-1\rangle ; \mathbb{Q}),
\end{aligned}
$$




$$
\begin{aligned}
& C_{1}=E_{2}^{0, a+b-2} \cong H^{a+b-2}(W\langle b-1\rangle ; \mathbb{Q}), \\
& C_{2}=E_{2}^{0, a+b-1} \cong H^{a+b-1}(W\langle b-1\rangle ; \mathbb{Q}) .
\end{aligned}
$$

The arrows represent the first few differentials that are not automatically trivial because either their target or domain is a zero group. The unlabelled groups at the positions indicated by the solid black circles are

$$
E_{2}^{a, b-1} \cong A_{1} \otimes B_{1}, \quad E_{2}^{a, b} \cong A_{1} \otimes B_{2} \quad \text { and } \quad E_{2}^{a+1, b-1} \cong A_{2} \otimes B_{1} .
$$

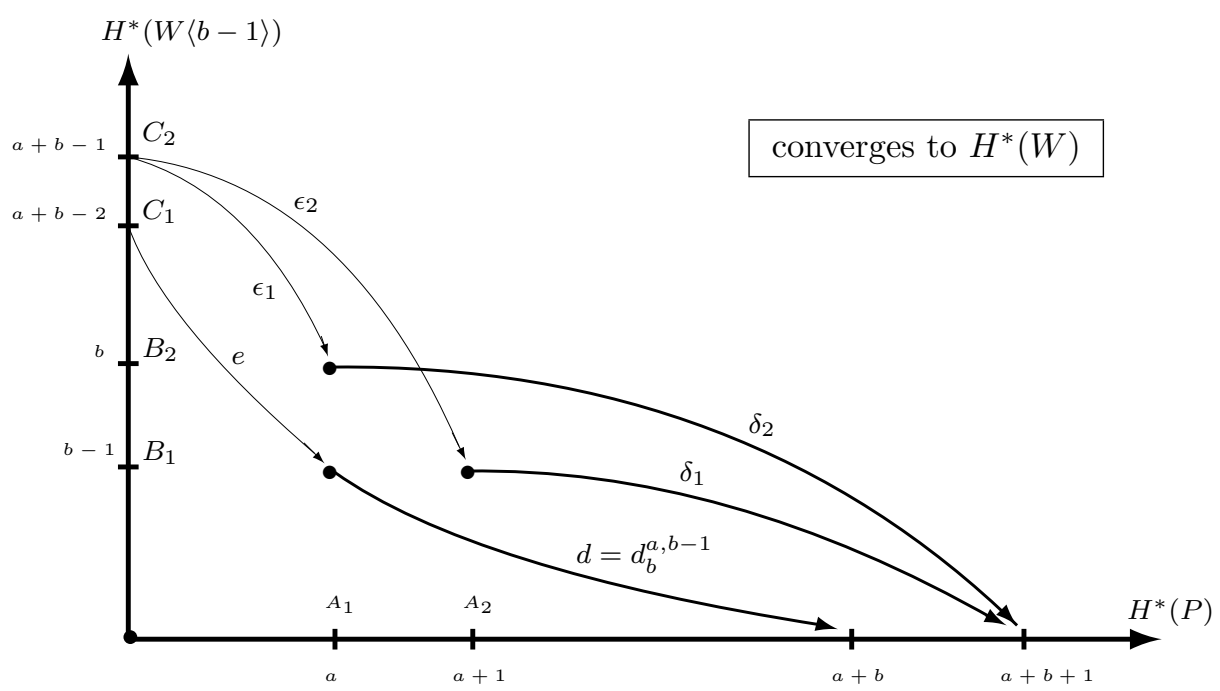

Figure 1: The relevant differentials.

Proposition 6.1. The Leray-Serre spectral sequence of Figure 1 constrains the first three terms of the categorical sequence $\sigma_{P}$ as follows:

1. If $d: A_{1} B_{1} \rightarrow H^{a+b}(P)$ is nonzero, then $\sigma_{P}=(a, b, a+b, \ldots)$.

2. If $\delta_{1}: A_{2} B_{1} \rightarrow H^{a+b+1}(P)$ is nonzero, then there is a nonzero class in $H^{a+b+1}(P)$ with category weight 3 , and hence $\sigma_{P}(3) \leqslant a+b+1$.

3. If $\delta_{1}=0$, then $\delta_{2}$ is a map $A_{1} B_{2} \rightarrow H^{a+b+1}(P)$; under this assumption, if $\delta_{2} \neq 0$, then there is a nonzero class in $H^{a+b+1}(P)$ with category weight 3 , and hence $\sigma_{P}(3) \leqslant a+b+1$.

Proof. The image of $d_{r}$ in $E_{r}^{*, 0}$ is zero for $r<b-1$, which shows that $E_{b-1}^{*, 0} \cong H^{*}(P)$ as algebras. Since $H^{k}(W)=0$ for all $k \geqslant b-1$, the differential $d_{b}: B_{1} \rightarrow H^{b}(P)$ must be an isomorphism.

Since $d_{r}\left(A_{1}\right)=0$ for all $r$, the fact that each spectral sequence differential $d_{r}$ is a derivation implies the commutativity of the square

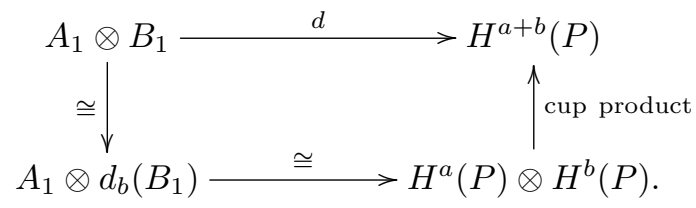


This shows that $d$ is nonzero if and only if the indicated cup product is nonzero. Together with Proposition 4.6, this proves (1).

If $d \neq 0$, then (2) follows immediately from (1), so we may assume that $d=0$. Under this assumption we can identify $E_{b}^{a+b+1,0}$ with $H^{a+b+1}(P)$ and fit the differential $\delta_{1}$ into the commutative square

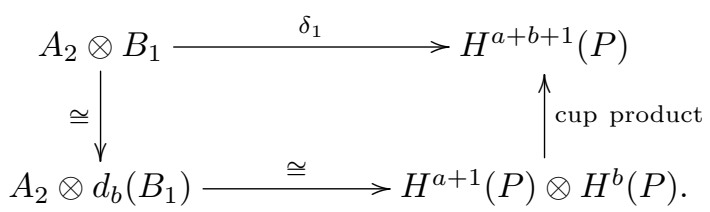

Now if $\delta_{1} \neq 0$, then the indicated cup product is nonzero. Since any class in dimension $\geqslant b$ has category weight $\geqslant 2$ by Proposition 4.5 , every nonzero product in dimension $a+b+1$ will have category weight $\geqslant 3$; then cat $\left(P_{a+b+1}\right) \geqslant 3$ and $\sigma_{P}(3) \leqslant a+b+1$ by Proposition 2.2.

Part (3) follows from a similar argument under the additional assumption that $d=0$ and $\delta_{1}=0$.

Proposition 6.2. For $n<2 b-3, H^{n}(W\langle b-1\rangle$; $\mathbb{Q}) \cong \operatorname{Hom}_{\mathbb{Q}}\left(\pi_{n}(W), \mathbb{Q}\right)$.

Proof. Since the proposition only makes claims about groups in the stable range, we can replace $W\langle b-1\rangle$ with a wedge of rational spheres $U$. For wedges of rational spheres, the Hurewicz map $\pi_{k}(U) \rightarrow H_{k}(U ; \mathbb{Q})$ is a split surjection in all dimensions and an isomorphism in the stable range. Therefore

$$
\begin{aligned}
H^{n}(W\langle b-1\rangle) & \cong H^{n}(U) \cong \operatorname{Hom}\left(H_{n}(U), \mathbb{Q}\right) \cong \operatorname{Hom}\left(\pi_{n}(U), \mathbb{Q}\right) \\
& \cong \operatorname{Hom}\left(\pi_{n}(W), \mathbb{Q}\right) .
\end{aligned}
$$

\subsection{Counting dimensions}

The dimensions of the vector spaces $V_{n}$ are determined using the following formulas. These formulas are concerned with the basis for a free graded Lie algebra $L$ given by the 'basic products', which are certain bracket monomials in the generators. Since all bases for a given vector space have the same cardinality, these results apply equally well to our chosen basis $\mathcal{B}$.

Theorem 6.3 (Witt). Let $L$ be the free graded Lie algebra on generators $x_{1}, x_{2}$, $\ldots, x_{k}$. The number of basic products involving $x_{i}$ exactly $n_{i}$ times is

$$
\frac{1}{n} \sum_{d \mid n_{0}} \mu(d) \frac{\left(\frac{n}{d}\right) !}{\left(\frac{n_{1}}{d}\right) !\left(\frac{n_{2}}{d}\right) ! \cdots\left(\frac{n_{k}}{d}\right) !},
$$

where $\mu$ is the Möbius function, $n_{0}=\operatorname{gcd}\left\{n_{1}, n_{2}, \ldots, n_{k}\right\}$ and $n=n_{1}+n_{2}+\cdots+n_{k}$.

Corollary 6.4. Let $W=S^{n_{1}} \vee S^{n_{2}} \vee \cdots \vee S^{n_{k+1}}$. The number of basic products with $s$ factors taken from $\left\{x_{1}, x_{2}, \ldots, x_{k}\right\}$ and $t$ factors of $x_{k+1}$ is given by the formula

$$
\frac{1}{s+t} \sum_{d \mid t} \mu(d)\left(\begin{array}{c}
(s+t) / d \\
s / d
\end{array}\right) k^{\frac{s}{d}} .
$$


Proof. Let $W=S^{n_{1}} \vee S^{n_{2}} \vee \cdots \vee S^{n_{k+1}}$. The Hilton-Milnor theorem tells us that

$$
\pi_{*}(W) \cong \bigoplus_{\beta \in \mathcal{B}} \pi_{*}\left(S^{|\beta|+1}\right)
$$

where $\mathcal{B}$ is a basis for the free Lie algebra $L\left(y_{1}, y_{2}, \ldots, y_{k+1}\right)$ and each $\left|y_{i}\right|=n_{i}-1$. We apply the Witt formula to count the number of basic products with $s$ factors taken from $\left\{x_{1}, x_{2}, \ldots, x_{k}\right\}$ and $t$ factors of $x_{k+1}$.

To simplify notation in our calculation, we write $\bar{s}=\left(s_{1}, s_{2}, \ldots, s_{k}\right)$ and $|\bar{s}|=$ $\sum s_{i}=s$. Further, $d \mid \bar{s}$ indicates that $d \mid s_{i}$ for each $i$ and $(\bar{s}, t)$ denotes the greatest common divisor of $s_{1}, s_{2}, \ldots, s_{k}$ and $t$.

Now the formula of Theorem 6.3 tells us that the number of basic products with $s_{i}$ factors of $x_{i}$ for $i=1,2, \ldots, k$ is

$$
\frac{1}{s+t} \sum_{d \mid(\bar{s}, t)} \mu(d) \frac{\left(\frac{s+t}{d}\right) !}{\left(\frac{s_{1}}{d}\right) !\left(\frac{s_{2}}{d}\right) ! \cdots\left(\frac{s_{k}}{d}\right) !\left(\frac{t}{d}\right) !} .
$$

We sum these expressions over all $\bar{s}$ with $|\bar{s}|=s$ as follows:

$$
\begin{aligned}
& \frac{1}{s+t} \sum_{|\bar{s}|=s} \sum_{d \mid(\bar{s}, t)} \mu(d) \frac{\left(\frac{s+t}{d}\right) !}{\left(\frac{s_{1}}{d}\right) !\left(\frac{s_{2}}{d}\right) ! \cdots\left(\frac{s_{k}}{d}\right) !\left(\frac{t}{d}\right) !} \\
& =\frac{1}{s+t} \sum_{d \mid t} \frac{\mu(d)\left(\frac{s+t}{d}\right) !}{\left(\frac{t}{d}\right) !} \sum_{\substack{d|\bar{s}\\
| \bar{s} \mid=s}} \frac{1}{\left(\frac{s_{1}}{d}\right) !\left(\frac{s_{2}}{d}\right) ! \cdots\left(\frac{s_{k}}{d}\right) !} \\
& =\frac{1}{s+t} \sum_{d \mid t} \frac{\mu(d)\left(\frac{s+t}{d}\right) !}{\left(\frac{t}{d}\right) !\left(\frac{s}{d}\right) !} \sum_{\substack{d|\vec{s}\\
| \bar{s} \mid=s}} \frac{\left(\frac{s}{d}\right) !}{\left(\frac{s_{1}}{d}\right) !\left(\frac{s_{2}}{d}\right) ! \cdots\left(\frac{s_{k}}{d}\right) !} \\
& =\frac{1}{s+t} \sum_{d \mid t} \mu(d)\left(\begin{array}{c}
(s+t) / d \\
s / d
\end{array}\right) \sum_{\substack{d|\bar{s}\\
| \bar{s} \mid=s}} \frac{\left(\frac{s}{d}\right) !}{\left(\frac{s_{1}}{d}\right) !\left(\frac{s_{2}}{d}\right) ! \cdots\left(\frac{s_{k}}{d}\right) !} \\
& =\frac{1}{s+t} \sum_{d \mid t} \mu(d)\left(\begin{array}{c}
(s+t) / d \\
s / d
\end{array}\right)(\overbrace{1+1+\cdots+1}^{k})^{\frac{s}{d}} \\
& =\frac{1}{s+t} \sum_{d \mid t} \mu(d)\left(\begin{array}{c}
(s+t) / d \\
s / d
\end{array}\right) k^{\frac{s}{d}} \text {. }
\end{aligned}
$$

Next we establish the dominant terms for the dimensions of the vector spaces involved in our computation, viewed as functions of $k$.

Proposition 6.5. Let $W=\left(\bigvee_{1}^{k} S^{a}\right) \vee S^{a+1}$. Write $n=s(a-1)+t a+1$ with $s, t \geqslant 0$ and $s$ as large as possible. Then

$$
\operatorname{dim}\left(\pi_{n}(W)\right)=\frac{k^{s}}{s+t}\left(\begin{array}{c}
s+t \\
s
\end{array}\right)+\text { lower order terms }
$$

as a function of $k$. If $n$ cannot be decomposed in the required fashion, then $\pi_{n}(W)=0$.

Proof. We prove this by induction on $n$, the statement being obvious in the case $n=a$ (so $s=1$ and $t=0$ ). Supposing the growth estimate to be valid for all values 
less than $n$, we address the question at hand: the dimension of $\pi_{n}(W)$. Rationally,

$$
\pi_{*}\left(S^{m}\right)= \begin{cases}\Sigma^{m} \mathbb{Q} \cdot \iota_{m} & \text { if } m \text { is odd } \\ \Sigma^{m} \mathbb{Q} \cdot \iota_{m} \oplus \Sigma^{2 m-1} \mathbb{Q} \cdot\left[\iota_{m}, \iota_{m}\right] & \text { if } m \text { is even. }\end{cases}
$$

Depending on the parity of $n$, therefore, the dimension of $\pi_{n}(W)$ is either

- the total number of basic products of dimension $n$, if $n$ is even, or

- the total number of basic products of dimension $n$, plus the number of basic products of dimension $\frac{n+1}{2}$, if $n$ is odd.

Write $n=s(a-1)+t a+1$ with $s$ as large as possible while $t \geqslant 0$. The basis for $\pi_{n}(W)$ can be divided into three subsets. First of all, and most importantly, there are the Whitehead product monomials that are not Whitehead product squares and have $s$ factors of dimension $a$ and $t$ factors of dimension $a+1$; Corollary 6.4 shows that the number of such monomials, viewed as a function of $k$ with $n, s$ and $t$ held fixed, is

$$
\frac{k^{s}}{s+t}\left(\begin{array}{c}
s+t \\
s
\end{array}\right)+\text { lower order terms. }
$$

Secondly, we have the Whitehead product monomials which are not squares and have $s^{\prime}$ factors of dimension $a$ and $t^{\prime}$ factors of dimension $a+1$, with $s^{\prime}<s$. Applying Corollary 6.4 again, we see that the number of such monomials is

$$
\frac{k^{s^{\prime}}}{s^{\prime}+t^{\prime}}\left(\begin{array}{c}
s^{\prime}+t^{\prime} \\
s^{\prime}
\end{array}\right)+\text { lower order terms }
$$

Finally, there might be some bracket squares. The number of these will be exactly the same as the number of basic products in dimension $\frac{1}{2}(n+1)$. If we write $\frac{1}{2}(n+1)=$ $s^{\prime \prime}(a-1)+t^{\prime \prime} a+1$ where $s^{\prime \prime}$ is as large as possible with $t^{\prime \prime} \geqslant 0$, then by the induction hypothesis the number of such terms is

$$
\frac{k^{s^{\prime \prime}}}{s^{\prime \prime}+t^{\prime \prime}}\left(\begin{array}{c}
s^{\prime \prime}+t^{\prime \prime} \\
s^{\prime \prime}
\end{array}\right)+\text { lower order terms. }
$$

Therefore the dimension of $\pi_{n}(W)$ is

$$
\frac{k^{s}}{s+t}\left(\begin{array}{c}
s+t \\
s
\end{array}\right)+\frac{k^{s^{\prime}}}{s^{\prime}+t^{\prime}}\left(\begin{array}{c}
s^{\prime}+t^{\prime} \\
s^{\prime}
\end{array}\right)+\frac{k^{s^{\prime \prime}}}{s^{\prime \prime}+t^{\prime \prime}}\left(\begin{array}{c}
s^{\prime \prime}+t^{\prime \prime} \\
s^{\prime \prime}
\end{array}\right)+\text { lower order terms } .
$$

Notice that the equation $\frac{1}{2}(n+1)=s^{\prime \prime}(a-1)+t^{\prime \prime} a+1$ implies $n=2 s^{\prime \prime}(a-1)+$ $2 t^{\prime \prime} a+1$; since $t^{\prime \prime} \geqslant 0,2 t^{\prime \prime} \geqslant 0$, and since $s$ is the largest number fitting into such an equation, we have $s \geqslant 2 s^{\prime \prime}>s^{\prime \prime}$. Since we have $s>s^{\prime}$ by definition, the dominant term in $\operatorname{dim}_{\mathbb{Q}}\left(\pi_{n}(W)\right)$ as a function of $k$ is the first term $\frac{k^{s}}{s+t}\left(\begin{array}{c}s+t \\ s\end{array}\right)$, as required.

\subsection{Realizing $(a, b, a+b, \ldots)$}

We show that sequences of the form $(a, b, a+b, \ldots)$ are realizable if $b \equiv 2 \bmod a-1$. Writing $W(k)=\bigvee_{1}^{k} S^{a}$ and $P(k)=P_{b-1}(W(k))$. Our argument uses the dimension estimate of Proposition 6.5 to show that $P(k)$ realizes the sequence if $k$ is large enough. 
Since $b \equiv 2 \bmod a-1$, we can write

$$
b-1=s \cdot(a-1)+0 \cdot a+1,
$$

for some $s$. Clearly the displayed expression for $b-1$ has $s$ as large as possible (with nonnegative coefficient of $a$ ), so, referring to Figure 1 and applying Proposition 6.5, we have

$$
\begin{aligned}
& \operatorname{dim}\left(A_{1}\right)=k, \\
& \operatorname{dim}\left(B_{1}\right)=\frac{1}{s} k^{s}+\text { lower order terms }
\end{aligned}
$$

and therefore

$$
\operatorname{dim}\left(A_{1} B_{1}\right)=\frac{1}{s} k^{s+1}+\text { lower order terms. }
$$

On the other hand

$$
\operatorname{dim}\left(C_{1}\right)=\frac{1}{s+1} k^{s+1}+\text { lower order terms }
$$

and it follows that $\operatorname{dim}\left(A_{1} B_{1}\right)>\operatorname{dim}\left(C_{1}\right)$ for $k$ large enough. Since $H^{a+b-1}(W(k))=$ 0 , the sequence

$$
C_{1} \stackrel{e}{\rightarrow} A_{1} B_{1} \stackrel{d}{\rightarrow} H^{a+b}(P(k))
$$

in Figure 1 is exact. Choosing $k$ large enough that $\operatorname{dim}\left(A_{1} B_{1}\right)>\operatorname{dim}\left(C_{1}\right)$, we find that the differential $d$ is nonzero, and Proposition 6.1 ensures that $\sigma_{P(k)}=(a, b, a+b, \ldots)$. This completes the proof of Theorem 1.1.

6.4. Realizing $(a, b, c, \ldots)$

It remains to show that superadditive sequences of the form $(a, b, c, \ldots)$ are realizable provided $2 a \leqslant b<c-a$ and $s(a-1)+t a+2=c$ can be solved with $s, t \in \mathbb{N}$.

Lemma 6.6. If $(a, b, c, \ldots)$ is (rationally) realizable then so is $\left(a, b^{\prime}, c, \ldots\right)$ for any $b^{\prime}$ such that $2 a \leqslant b^{\prime} \leqslant b$.

Proof. Let $Z$ be a space such that $\sigma_{Z}=(a, b, c, \ldots)$. Write $b^{\prime}=a+a^{\prime}$, so that $a^{\prime} \geqslant a$ since $b^{\prime} \geqslant 2 a$. We can see that the space $Z \vee\left(S^{a} \times S^{a^{\prime}}\right)$ has the required sequence by applying the formula

$$
\sigma_{X \vee Y}(k)=\min \left\{\sigma_{X}(k), \sigma_{Y}(k)\right\},
$$

established for all spaces in [13, Prop. 3.1], and using the elementary computation $\sigma_{S^{a} \times S^{a^{\prime}}}=\left(a, a+a^{\prime}, \infty, \ldots\right)[\mathbf{1 3}$, Cor. 3.3].

Because of Lemma 6.6, it suffices to show that whenever $c \geqslant 3 a+1$, the sequence $(a, c-(a+1), c, \ldots)$ is rationally realizable. Write $b=c-(a+1)$.

If $c-a \equiv 2 \bmod a-1$ then the realizability result established in Section 6.3 shows that $(a, c-a, c, \ldots)$ is rationally realizable, and we are done by Lemma 6.6. Thus we may assume from now on that

$$
b=(c-a)-1 \not \equiv 1 \bmod a-1,
$$

which is to say $b \not \equiv 1 \bmod a-1$.

Our task is to produce a space $Q$ with $\sigma_{Q}=(a, b, a+b+1, \ldots)$. 
Adapting the method of Section 6.3, we write $W(k)=\left(\bigvee_{1}^{k} S^{a}\right) \vee S^{a+1}$, and consider the fiber sequence

$$
W(k)\langle b-1\rangle \rightarrow W(k) \rightarrow P_{b-1}(W(k)),
$$

and agree to write $P(k)=P_{b-1}(W(k))$ from now on. Evidently $\sigma_{P(k)}(2) \geqslant b$, so it suffices (by Lemma 6.6) to show that $\sigma_{P(k)}(3)=c$. By superadditivity we have

$$
\sigma_{P(k)}(3) \geqslant \sigma_{P(k)}(2)+\sigma_{P(k)}(1) \geqslant b+a=c-1 .
$$

Let $A_{1}, A_{2}, B_{1}, B_{2}$ and $C_{2}$ be the vector spaces indicated in Figure 1 ; these vector spaces and their dimensions are functions of $k$. To estimate these dimensions, write

$$
b=s(a-1)+t a+1 \quad \text { with } s, t \geqslant 0 \text { and } s \text { as large as possible. }
$$

Since $b \not \equiv 1 \bmod a-1$ we must have $0<t<a-1$; thus

$$
b-1=(s+1)(a-1)+(t-1) a+1,
$$

with $s+1, t-1 \geqslant 0$ and $t-1$ as small as possible. Finally, we get

$$
b+(a-1)=(s+1)(a-1)+t a+1,
$$

with $t$ as small as possible with $t \geqslant 0$. Therefore Proposition 6.5 gives:

$$
\begin{aligned}
\operatorname{dim}\left(A_{1}\right) & =k, \\
\operatorname{dim}\left(A_{2}\right) & =1, \\
\operatorname{dim}\left(B_{1}\right) & =\frac{1}{(s+1)+(t-1)} k^{s+1}\left(\begin{array}{c}
(s+1)+(t-1) \\
s+1
\end{array}\right)+\text { lower order terms }, \\
\operatorname{dim}\left(B_{2}\right) & =\frac{1}{s+t} k^{s}\left(\begin{array}{c}
s+t \\
s
\end{array}\right)+\text { lower order terms }, \\
\operatorname{dim}\left(C_{2}\right) & =\frac{1}{(s+1)+t} k^{s+1}\left(\begin{array}{c}
(s+1)+t \\
s+1
\end{array}\right)+\text { lower order terms } .
\end{aligned}
$$

The dominant term in the expression $\operatorname{dim}\left(A_{1}\right) \operatorname{dim}\left(B_{2}\right)+\operatorname{dim}\left(A_{2}\right) \operatorname{dim}\left(B_{1}\right)$ (considered as a function of $k$ ) is

$$
k \cdot \frac{1}{s+t} k^{s}\left(\begin{array}{c}
s+t \\
s
\end{array}\right)+1 \cdot \frac{1}{s+t} k^{s+1}\left(\begin{array}{c}
s+t \\
s+1
\end{array}\right)=1 \cdot \frac{1}{s+t} k^{s+1}\left(\begin{array}{c}
s+t+1 \\
s+1
\end{array}\right),
$$

while the dominant term in $\operatorname{dim}\left(C_{2}\right)$ is

$$
\frac{1}{s+t+1} k^{s+1}\left(\begin{array}{c}
s+t+1 \\
s+1
\end{array}\right) \text {. }
$$

It follows that the inequality

$$
\operatorname{dim}\left(A_{1}\right) \operatorname{dim}\left(B_{2}\right)+\operatorname{dim}\left(A_{2}\right) \operatorname{dim}\left(B_{1}\right)>\operatorname{dim}\left(C_{2}\right)
$$

holds for $k$ large enough.

So let us choose a large enough $k$ and write $W=W(k)$ and $P=P(k)$. Since $H^{a+b}(W)=0$, the sequences

$$
C_{2} \stackrel{\epsilon_{2}}{\longrightarrow} A_{2} B_{1} \stackrel{\delta_{1}}{\longrightarrow} H^{a+b+1}(P)
$$

and 


$$
\operatorname{ker}\left(\epsilon_{2}\right) \stackrel{\epsilon_{1}}{\longrightarrow} A_{1} B_{2} \stackrel{\delta_{2}}{\longrightarrow} \operatorname{coker}\left(\delta_{1}\right)
$$

from Figure 1 are exact. If the differentials $\delta_{1}$ and $\delta_{2}$ were both zero, then

$$
\operatorname{dim}\left(C_{2}\right) \geqslant \operatorname{dim}\left(A_{2}\right) \operatorname{dim}\left(B_{1}\right)+\operatorname{dim}\left(A_{1}\right) \operatorname{dim}\left(B_{2}\right),
$$

contradicting the inequality $(\star)$. Therefore, we may apply Proposition 6.1 to deduce that there is a nonzero element $u \in H^{a+b+1}(P)$ with $\operatorname{wgt}(u)=3$.

Since $P$ is a simply-connected rational space, we may give its $(a+b+1)$-skeleton $P_{a+b+1}$ a rational CW decomposition which is also a homology decomposition [2]. The tail end of this decomposition gives us two cofiber sequences

$$
M_{a+b-1} \stackrel{\alpha}{\rightarrow} P_{a+b-1} \stackrel{i}{\rightarrow} P_{a+b} \quad \text { and } \quad M_{a+b} \stackrel{\beta}{\rightarrow} P_{a+b} \rightarrow P_{a+b+1},
$$

where $M_{a+b-1} \simeq \bigvee S^{a+b-1}$ and $M_{a+b} \simeq \bigvee S^{a+b}$ (rationally). Recall that part of the definition of a homology decomposition is that the attaching maps $\alpha$ and $\beta$ induce 0 on homology.

We show that the attaching map $\beta$ factors up to homotopy through the inclusion $i: P_{a+b-1} \rightarrow P_{a+b}$ of the (rational) $(a+b-1)$-skeleton into the $(a+b)$-skeleton. Consider the diagram

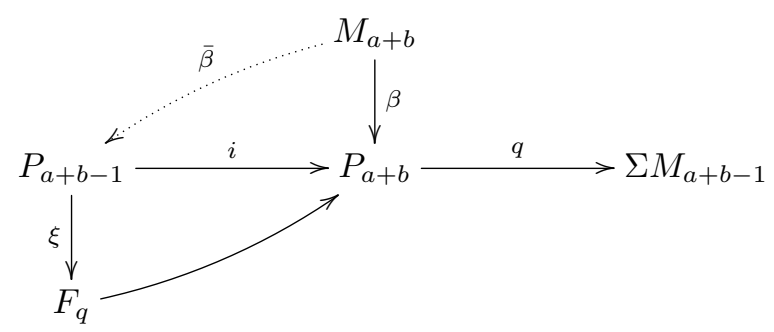

in which $F_{q}$ is the homotopy fiber of the quotient map $q$. The map $q \circ \beta: M_{a+b} \rightarrow$ $\Sigma M_{a+b-1}$ is a map from one wedge of rational spheres of dimension $a+b$ to another, and it induces zero on homology because $\beta$ does. Therefore $q \circ \beta \simeq *$ and it follows that $\beta$ factors up to homotopy through $F_{q}$. Now $P_{a+b-1}$ is $(a-1)$-connected and $\Sigma M_{a+b-1}$ is $(a+b-1)$-connected. Since

$$
(a+b-1)+(a-1)=a+b+(a-2) \geqslant a+b,
$$

the Blakers-Massey theorem ensures that $\xi$ is an $(a+b)$-equivalence. This implies that the dotted arrow $\bar{\beta}$ exists making the diagram commute up to homotopy.

Now form the space $Q=P_{c-2} \cup_{\bar{\beta}} C M_{a+b}$, which fits into the diagram

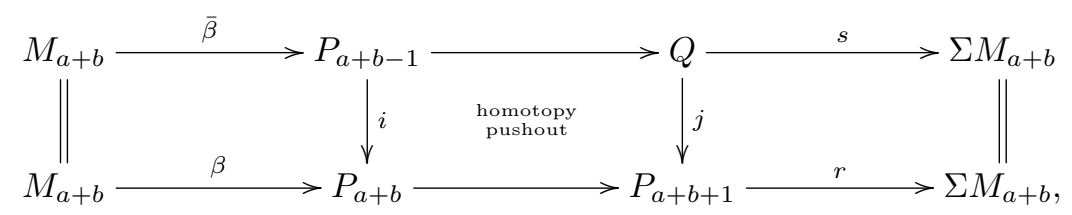

whose rows are cofiber sequences. Since the center square is a homotopy pushout square, the cofiber of $j$ is homotopy equivalent to the cofiber of $i$. This common cofiber is $\Sigma M_{a+b-1}$, which is $(a+b-1)$-connected. Since $a \geqslant 2$ it follows that $j$ is a $(b+1)$-equivalence and therefore $\sigma_{Q}(2)=\sigma_{P_{a+b+1}}(2)=b$. 
Since $\beta$ and $\bar{\beta}$ are zero on homology, $r$ and $s$ induce isomorphisms on $H^{a+b+1}$. We've shown that there is an element $u \in H^{a+b+1}(P)$ with $\operatorname{wgt}(u)=3$. Then $v=$ $j^{*}\left(\left.u\right|_{P_{a+b+1}}\right) \in H^{a+b+1}(Q)$ is nonzero and $\operatorname{wgt}(v) \geqslant 3$, so $a+b \leqslant \sigma_{Q}(3) \leqslant a+b+1$. The crucial difference between $P$ and $Q$ is that $H^{a+b}(Q)=0$ and so it is impossible for $\sigma_{Q}(3)$ to be equal to $a+b$. Therefore

$$
\sigma_{Q}=(a, b, a+b+1, \infty, \ldots),
$$

which completes the proof of Theorem 1.2(b).

\section{References}

[1] R. Bott and H. Samelson. On the Pontryagin product in spaces of paths. Comment. Math. Helv., 27:320-337 (1954), 1953.

[2] E.H. Brown, Jr. and A.H. Copeland, Jr. An homology analogue of Postnikov systems. Michigan Math. J, 6:313-330, 1959.

[3] F.R. Cohen, J.C. Moore, and J.A. Neisendorfer. The double suspension and exponents of the homotopy groups of spheres. Ann. of Math. (2), 110(3):549$565,1979$.

[4] O. Cornea, G. Lupton, J. Oprea, and D. Tanré. Lusternik-Schnirelmann Category, volume 103 of Math. Surveys Monogr. Amer. Math. Soc., Providence, RI, 2003.

[5] A.N. Dranishnikov, M.G. Katz, and Y.B. Rudyak. Small values of the Lusternik-Schnirelman category for manifolds. Geom. Topol., 12(3):1711-1727, 2008. "Schnirelman" in title changed to "Schnirelmann" on journal site.

[6] E. Fadell and S. Husseini. Category weight and Steenrod operations. Bol. Soc. Mat. Mex. (2), 37(1-2):151-161, 1992. Papers in honor of José Adem (in Spanish).

[7] Y. Félix, S. Halperin, and J.-C. Thomas. Rational Homotopy Theory, volume 205 of Grad. Texts in Math. Springer-Verlag, New York, 2001.

[8] T. Ganea. A generalization of the homology and homotopy suspension. Comment. Math. Helv., 39:295-322, 1965.

[9] P. Ghienne. The Lusternik-Schnirelmann category of spaces in the Mislin genus of $\mathrm{Sp}(3)$. In Lusternik-Schnirelmann Category and Related Topics (South Hadley, MA, 2001), volume 316 of Contemp. Math., pages 121-126. Amer. Math. Soc., Providence, RI, 2002.

[10] J.D. Houck. A Pattern in the Lusternik-Schnirelmann Category of Rational spaces. ProQuest LLC, Ann Arbor, MI, 2013. Thesis (Ph.D.), Western Michigan University.

[11] I.M. James. On category, in the sense of Lusternik-Schnirelmann. Topology, 17(4):331-348, 1978.

[12] J.W. Milnor and J.C. Moore. On the structure of Hopf algebras. Ann. of Math. (2), 81:211-264, 1965.

[13] R. Nendorf, N. Scoville, and J. Strom. Categorical sequences. Algebr. Geom. Topol., 6:809-838, 2006. 
[14] Y.B. Rudyak. On category weight and its applications. Topology, 38(1):37-55, 1999.

[15] P.A. Schweitzer. Secondary cohomology operations induced by the diagonal mapping. Topology, 3:337-355, 1965.

[16] J. Strom. Lusternik-Schnirelmann category of spaces with free fundamental group. Algebr. Geom. Topol., 7:1805-1808, 2007.

[17] J. Strom. Essential category weight and phantom maps. In Cohomological Methods in Homotopy Theory (Bellaterra, 1998), volume 196 of Progr. Math., pages 409-415. Birkhäuser, Basel, 2001.

[18] J.A. Strom. Category Weight and Essential Category Weight. ProQuest LLC, Ann Arbor, MI, 1997. Thesis (Ph.D.), The University of Wisconsin-Madison.

[19] G.W. Whitehead. Elements of Homotopy Theory, volume 61 of Grad. Texts in Math. Springer-Verlag, New York, Berlin, 1978.

Julienne Dare Houck julie.dare.houck@gmail.com

Grand Rapids, MI, USA

Jeffrey Strom jeff.strom@wmich.edu

Department of Mathematics, Western Michigan University, Kalamazoo, MI 49008, USA 